\title{
Case report: A Novel Intergenic KIF5B-METFusion Variant in a Patient with Gastric Cancer
}

\author{
Zhiwei Wu \\ Yangzhou University \\ Yu Sha \\ Yangzhou University \\ Qing Chen \\ Yangzhou University \\ Juan Hou \\ Yangzhou University \\ Yan Sun \\ Yangzhou University \\ Wangkun Lu \\ Yangzhou University \\ Jing Chen \\ Yangzhou University \\ Lijiang Yu ( $\square$ yulijiang830@163.com ) \\ Yangzhou University https://orcid.org/0000-0002-0510-8542
}

\section{Case Report}

Keywords: KIF5B, MET, Gastric cancer, Case report

Posted Date: September 4th, 2020

DOI: https://doi.org/10.21203/rs.3.rs-60984/v1

License: (c) (1) This work is licensed under a Creative Commons Attribution 4.0 International License.

Read Full License 


\section{Abstract}

\section{Background}

MET fusion is a key driver mutation, but it is rare in gastric cancer (GC). Several MET inhibitors have been approved for the treatment of MET-positive patients, but the tumor response is heterogeneous. With the development of next-generation sequencing (NGS), diverse MET fusion partner genes have been identified. We reported a case of a fusion variant involving KIF5B-MET in GC.

Case presentation

After thoracoscopic inferior lobectomy plus lymph node dissection under general anesthesia, a "tumor within a tumor" was found in the lung tumor tissue of a 64-year-old nonsmoker male patient. Combining the medical history and the results of enzyme labeling, the focal area was considered to be GC. To seek potential therapeutic regimens, an intergenic region between KIF5B and MET fusion was identified. This fusion contains a MET kinase domain and coil-coiled domains encoded by KIF5B exons 1-25, which might drive oncogenesis.

\section{Conclusion}

Our founding could extend the spectrum and genomic landscape of MET fusions in GC and favor the development of personalized therapy.

\section{Background}

Gastric cancer (GC) is one of the most common malignancies. ${ }^{1}$ The incidence and mortality of gastric cancer vary by region, but more than $50 \%$ of cases occur in East Asia. ${ }^{2} \mathrm{GC}$ is responsible for $10.6 \%$ of new cases and $13.6 \%$ of cancer-related deaths in China. ${ }^{3}$ Targeted therapies designed to disrupt the activity of specific oncogenic signaling pathways have recently emerged as a promising therapeutic strategy in GC, such as the ToGA trial. Trastuzumab and ramucirumab improved the overall survival (OS) of patients by targeting the HER-2 and VEGFR genes. ${ }^{4,5}$

MET proto-oncogene receptor tyrosine kinase (MET) is a high-affinity proto-oncogene receptor tyrosine kinase that, upon activation, drives oncogenic pathways involved in cell proliferation, survival, and metastasis. As one of the major MET variants, MET fusion is a key driver mutation. The common fusion proteins are derived from chromosomal translocations, which are usually composed of $\mathrm{N}$-terminal dimerization domains provided by the kinase domains to which fusion partner proteins fuse to tyrosine kinases. These fusion mutations often lead to the activation of the kinase domain and provide ideal targets for the development of anticancer therapies. ${ }^{6}$ With the development of next-generation sequencing (NGS), diverse MET fusion partner genes, such as ETV6-MET, ${ }^{7} P T P R Z 1-M E T,{ }^{8}$ and CD74-MET, have been identified. ${ }^{9}$ However, $M E T$ gene fusion is a relatively rare but promising molecular target for 
individualized targeted therapies. Herein, we report a novel intergenic region between kinesin family member 5b (KIF5B) and MET fusion in a patient with GC.

\section{Case Presentation}

In April 2019, a 64-year-old nonsmoker male patient came to our hospital for further examination because chest computed tomography (CT) found nodules in the right lower lung two years ago. After admission, chest CT showed irregular patchy shadows in the lower lobe of the right lung, local thickening of the bronchial wall, and narrowing of the lumen, which was slightly denser than that 2 years ago (Fig. 1A). One week later, the patient underwent thoracoscopic inferior lobectomy plus lymph node dissection under general anesthesia. Postoperative pathology revealed that the heterotype glands in the focal area were significantly different from the main tumor body, the cytoplasm was rich in mucus, and most of the tumor cells were located in lymphatics (Fig. 1B). Combining the medical history and the results of enzyme labeling (Ki67 (+), D2-40 (+), MUC5AC (+) and Villin (+)) (Fig. 1C-F), it was considered that the focal area was GC. This lesion collided with the main tumor body, which was described as a "tumor within a tumor". The tumor size was $2.2 \times 1.5 \times 1.0 \mathrm{~cm}$.

To probe the genomic profile of the tumor for targeted therapy, the tissue specimens were subjected to NGS analysis, and an intergenic region between KIF5B and MET fusion was identified (Fig. 2A). The fusion of KIF5B-MET included exons 1-25 of KIF5B and exons 15-21 of MET, and the complete kinase structure of the MET protein was retained (Fig. 2B). The mutant allele frequency was $15.24 \%$. No other driver gene variants were found.

\section{Discussion And Conclusions}

MET fusions are rare in GC, and this is the first documented case of a patient with a MET fusion-positive tumor. In this study, we identified a patient with a fusion variant involving KIF5B-MET in GC. Our identified $K I F 5 B-M E T$ variant was the same as the most recently reported KIF5B-MET fusion variant in non-small cell lung cancer (NSCLC). ${ }^{10}$ This fusion gene was initially reported in one of 513 lung adenocarcinoma samples. ${ }^{11}$ Although several MET inhibitors have been approved for the treatment of MET-positive patients, the tumor response is heterogeneous, and the OS ranges from 1 to 36 months. ${ }^{10,12,13}$ One explanation is that the response of MET inhibitors to different MET fusion types differs. Other possible reasons include the existence of other driver mutations or primary resistance mutations in the kinase structure of the MET protein. Therefore, it seems necessary to comprehensively understand $M E T$ fusion information, and NGS could serve as a supplementary approach for MET status detection because of its high-throughput molecular analysis that can detect gene copy number alterations, deletions, insertions and fusions simultaneously.

To the best of our knowledge, this is the first report of breakpoints coexisting simultaneously in the intergenic region between the KIF5B gene and the MET gene, as well as a novel MET rearrangement in GC. This fusion contains a MET kinase domain and coil-coiled domains encoded by KIF5B exons 1-25, 
which might drive oncogenesis. Given the recent development of several MET inhibitors and their potential therapeutic efficacy for tumors expressing the KIF5B-MET fusion protein, we expect that further investigation of KIF5B-MET fusions will enable us to identify GC patients suitable for this targeted therapy.

\section{Abbreviations}

GC: Gastric cancer; MET: MET proto-oncogene receptor tyrosine kinase; NGS: next-generation sequencing; KIF5B: kinesin family member $5 b$; CT: computed tomography.

\section{Declarations}

\section{Ethics approval and consent to participate}

The study was performed in accordance with the Declaration of Helsinki and was approved by the Ethics Committee of the Jingjiang People's Hospital.

\section{Consent for publication}

A written informed consent was obtained from the patient for publication of his clinical details and clinical images in this case report.

\section{Availability of data and materials}

There is no supporting data available.

\section{Competing interest}

The authors declare no conflicts of interest.

\section{Funding}

None.

\section{Authors' contributions】}

YL and WZ designed the study, WZ, CQ and HJ drafted the manuscript. SY and CJ conducted the histological evaluation and immunohistochemical evaluation. SY and LW performed the NGS analysis. All authors read and approved the final manuscript.

\section{Acknowledgements}

None.

\section{References}


1. Torre LA, Bray F, Siegel RL, Ferlay J, Lortet-Tieulent J, Jemal A. Global cancer statistics, 2012. CA. Cancer. J Clin. 2015; 65:87-108. doi:10.3322/caac.21262

2. Wang FH, Shen L, Li J, Zhou ZW, Liang H, Zhang XT, et al. The Chinese Society of Clinical Oncology (CSCO): clinical guidelines for the diagnosis and treatment of gastric cancer. Cancer Commun (Lond). 2019; 39: 10. doi:10.1186/s40880-019-0349-9

3. Feng RM, Zong YN, Cao SM, Xu RH. Current cancer situation in China: good or bad news from the 2018 Global Cancer Statistics? Cancer Commun (Lond). 2019; 39: 22. doi:10.1186/s40880-0190368-6

4. Bang Y, Van Cutsem E, Feyereislova A et al. Trastuzumab in combination with chemotherapy versus chemotherapy alone for treatment of HER2-positive advanced gastric or gastro-oesophageal junction cancer (ToGA): a phase 3, openlabel, randomised controlled trial. Lancet. 2010; 376: 687-697.

5. Fuchs CS, Tomasek J, Yong CJ et al. Ramucirumab monotherapy for previously treated advanced gastric or gastro-oesophageal junction adenocarcinoma (REGARD): an international, randomised, multicentre, placebo-controlled, phase 3 trial. Lancet. 2014; 383: 31-39.

6. International Cancer Genome Consortium PedBrain Tumor Project. Recurrent MET fusion genes represent a drug target in pediatric glioblastoma. Nat Med. 2016;22(11):1314-1320. doi:10.1038/nm.4204

7. Rooper LM, Karantanos T, Ning Y, Bishop JA, Gordon SW, Kang H. Salivary Secretory Carcinoma With a Novel ETV6-MET Fusion: Expanding the Molecular Spectrum of a Recently Described Entity. Am J Surg Pathol. 2018;42(8):1121-1126. doi:10.1097/PAS.0000000000001065

8. Zeng AL, Yan W, Liu YW, et al. Tumour exosomes from cells harbouring PTPRZ1-MET fusion contribute to a malignant phenotype and temozolomide chemoresistance in glioblastoma. Oncogene. 2017;36(38):5369-5381. doi:10.1038/onc.2017.134

9. Pan Y, Zhang Y, Ye T, et al. Detection of Novel NRG1, EGFR, and MET Fusions in Lung Adenocarcinomas in the Chinese Population. J Thorac Oncol. 2019;14(11):2003-2008. doi:10.1016/j.jtho.2019.07.022

10. Gow CH, Liu YN, Li HY, et al. Oncogenic Function of a KIF5B-MET Fusion Variant in Non-Small Cell Lung Cancer. Neoplasia. 2018;20(8):838-847. doi:10.1016/j.neo.2018.06.007

11. Stransky N, Cerami E, Schalm S, Kim JL, Lengauer C. The landscape of kinase fusions in cancer. Nat Commun. 2014;5:4846. doi:10.1038/ncomms5846

12. Cho JH, Ku BM, Sun JM, et al. KIF5B-MET Gene Rearrangement with Robust Antitumor Activity in Response to Crizotinib in Lung Adenocarcinoma. J Thorac Oncol. 2018;13(3):e29-e31. doi:10.1016/j.jtho.2017.10.014

13. Plenker D, Bertrand M, de Langen AJ, et al. Structural Alterations of MET Trigger Response to MET Kinase Inhibition in Lung Adenocarcinoma Patients. Clin Cancer Res. 2018;24(6):1337-1343. doi:10.1158/1078-0432.CCR-17-3001

\section{Figures}



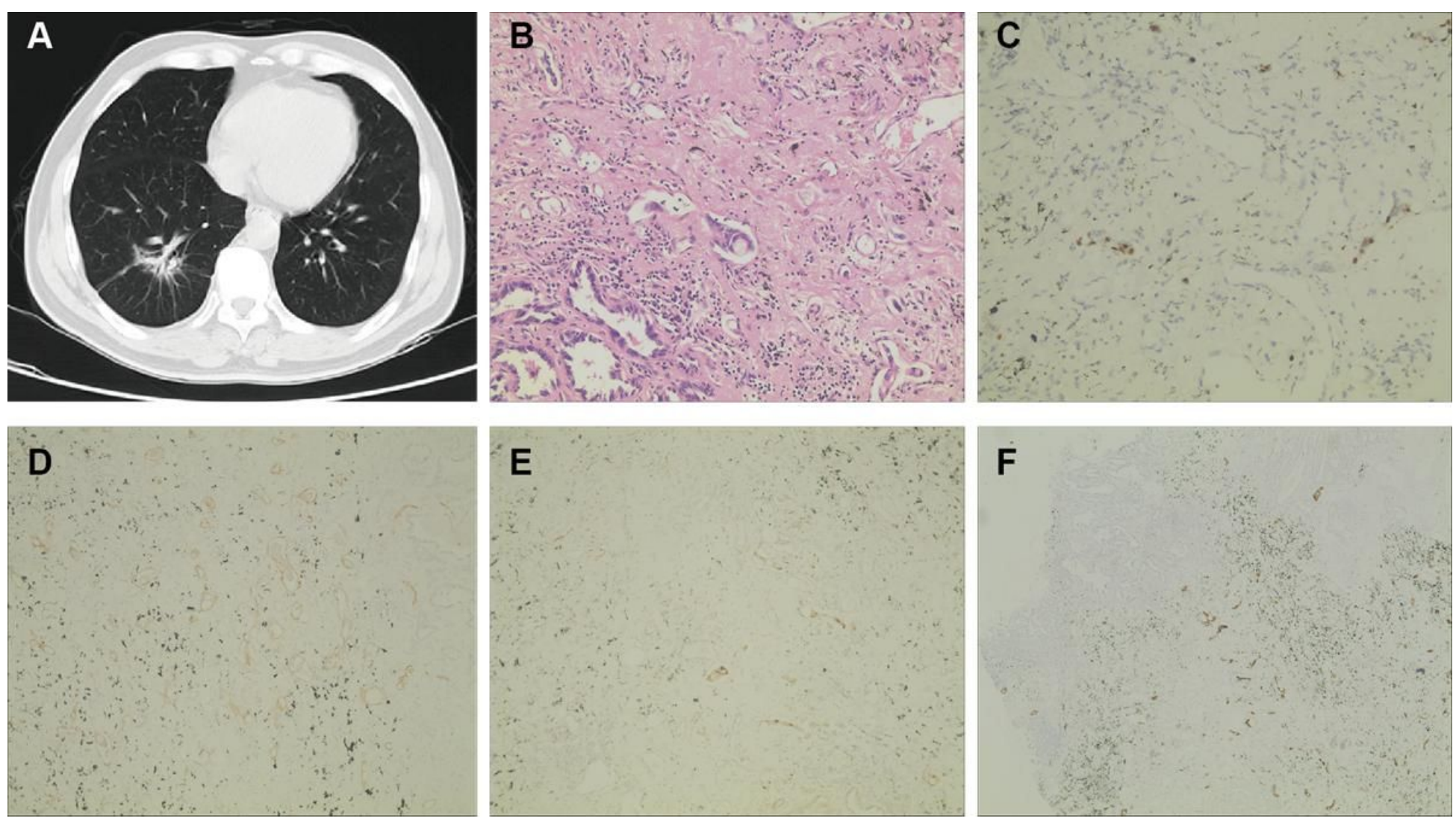

Figure 1

Patient disease diagnosis. A. CT diagnosis of lung tissue. B. Typical images of postoperative pathology. C-F. Immunohistochemistry staining images of Ki67 (+), D2-40 (+), MUC5AC (+) and Villin (+). 


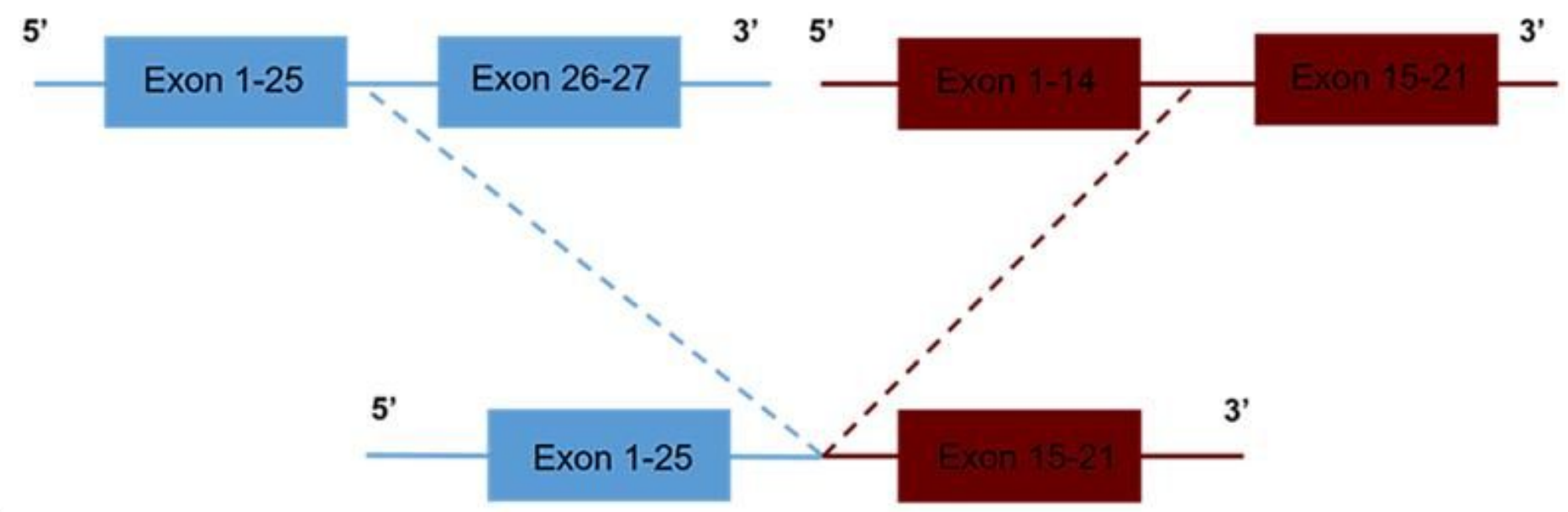

B

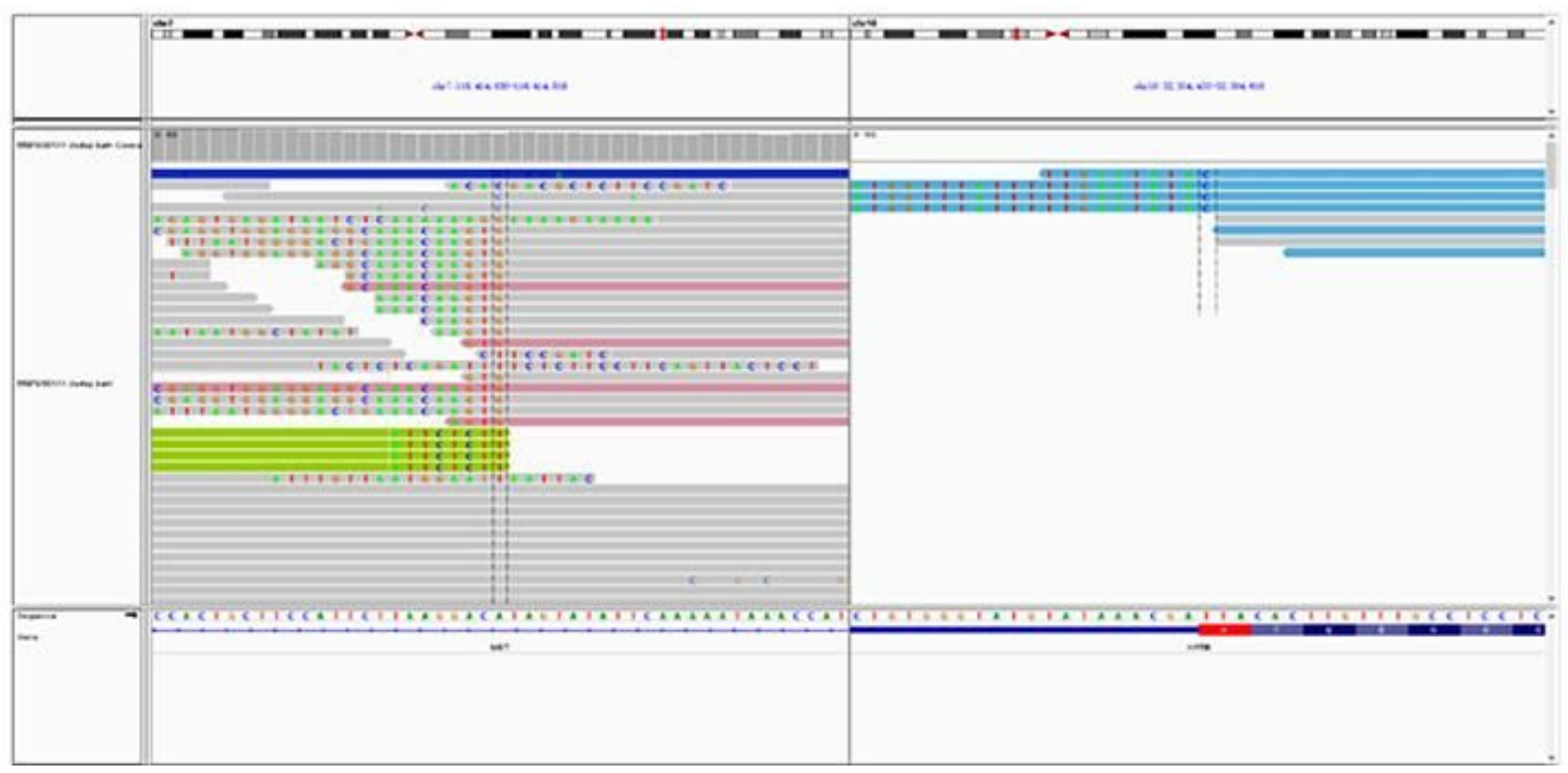

Figure 2

NGS findings for tumor tissue samples. A. A novel intergenic region between KIF5B exons 1-25 and the MET exon 15-21 fusion variant was identified. B. NGS results showing the breakpoint of the KIF5B-MET fusion.

\section{Supplementary Files}

This is a list of supplementary files associated with this preprint. Click to download.

- CAREchecklist.pdf 